\title{
Sclerotherapy with $3 \%$ polidocanol foam: revolutionizing outpatient treatment in patients with haemorrhoidal disease
}

\author{
Gaetano Gallo ${ }^{1}$ (1) $\cdot$ Maurizio Ronconi ${ }^{2} \cdot$ Mario Trompetto $^{3}$
}

Received: 9 February 2021 / Accepted: 16 February 2021 / Published online: 3 March 2021

(C) Italian Society of Surgery (SIC) 2021

\section{Dear Editor,}

We are very pleased to see how sclerotherapy (ST) for Haemorrhoidal Disease (HD) is claiming the attention of many experienced colorectal surgeons. In this context, the interest regarding ST was demonstrated by a recent article which confirmed its usefulness during the pandemic [1].

In particular, proctology was one of the most affected surgical specialties during the COVID-19 outbreak [2]. In fact, according to a recent worldwide survey including 1050 experts and aimed to evaluate the status of proctological practice, elective oncological and non-oncological surgery, emergency surgery and outpatient practice were reduced or fully stopped with an increased likelihood of delayed anorectal cancer. The outpatient practice was the least affected and 55\% (574) of the respondents continued these activities.

Daniel Zollikofer (St Gallen, Switzerland) first described in 1682 the use of a sclerosing agent to occlude veins by injection. Although no evidence has been reported, that was the flame that ignited the fire. After many years and several sclerosing agents, the success achieved in phlebology, with both liquids and foams, has led to the use of ST in the treatment of HD.

The so-called "modern" ST, or rather the use of foam, began with the randomized, controlled, single-blind trial published by Moser et al. [3]. The authors evaluated the efficacy and safety of polidocanol foam in comparison with liquid for the treatment of I degree HD. They demonstrated the superiority of the foam in terms of success rate ( $88 \%$ vs $69 \%$;

Gaetano Gallo

gallog@unicz.it

1 Department of Medical and Surgical Sciences, University of Catanzaro, Catanzaro, Italy

2 Department of General Surgery, ASST-Spedali Civili-Gardone V.T Hospital, Brescia, Italy

3 Department of Colorectal Surgery, S. Rita Clinic, Vercelli, Italy $p=0.01$ ), patient satisfaction ( 99 vs. $84 \% ; p=0.009$ ), number of sessions $(p<0.001)$ and amount of foam $(p<0.001)$ required to reach the success. Unfortunately, the 12-week follow-up is the main limitation of this trial.

Lisi et al. described their experience during the COVID19 pandemic in the treatment of 10 urgent cases with IIIand IV-degree HD [1]. The authors stated that "there are no studies concerning the use of foam in the treatment of third- and fourth-degree HD". We cannot agree with the authors. In fact, there are several studies in the literature, even if still very heterogeneous, which reported the results of polidocanol foam in the treatment of all stages of HD [4-6].

Ronconi et al. [4], in their experience with intraluminal injection of sclerosing foam, reported a total of 1427 procedures on 615 patients with an average of 2.32 ST sessions for patient and an average follow-up of 12 months.

The vast majority of patients had II- degree HD (317; $51.4 \%)$ and 17 (2.8\%), $253(41.1 \%)$ and 28 (4.7\%) had, respectively, I-, III- and IV-degree HD. Moreover, 97 patients had previous surgical excisional or non-excisional treatments for HD. Only 17 patients $(2.7 \%)$ experienced post-operative pain which has been solved conservatively.

Even if the authors carried out more than five ST sessions in 12 patients, in most of patients (83\%), one session was enough to solve the bleeding at all.

These results were consistent with a recent retrospective study [5], concerning the use of 3\% polidocanol foam in 66 patients with II- and III- degree HD, in which the overall success rate after a single session was $78.8 \%$ and only 9 patients experienced a median post-operative pain of 4 . There was no statistically significant difference between II- and III-degree HD in terms of success rate and complications. Furthermore, the success rate reached $86 \%$ after a second ST session.

Interestingly, 9 out 14 recurrences were successfully treated with de-arterialization and mucopexy pointing out the role of ST as a bridge to surgery approach. 
Another case series that considered the use of foam for II-IV degree HD was published by Fernandes and colleagues in 2019 [6]. The authors enrolled 2000 consecutive patients reporting a $98 \%$ success rate. However, there was a great variability in comparison with the above-mentioned studies concerning the size of the needle $(21 \mathrm{G})$ and both the percentage ( $2 \%$ polidocanol) and amount of foam used (10 or $20 \mathrm{ml}$ per session). Moreover, the use of a validated scoring system as well as of an adequate follow-up was lacking.

There are three other important concepts to be pointed out.

First, the diameter of the needle used for the injection and the amount of foam per piles must be mentioned.

Regarding the former, there is a huge difference based on the approach. For the endoscopic approach, a $23 \mathrm{G}$ needle [4] is often used while for the transanal approach, the ideal is a $20 \mathrm{G}$ needle [5].

As regards the amount of foam, according to the abovementioned studies [3-5], $2 \mathrm{cc}$ per pile with a maximum of 6-8 $\mathrm{cc}$ for the whole procedure is the best solution. We suggest producing a foam according to the Tessari method that has a ratio of $1.6 \mathrm{ml}$ of liquid polidocanol to $7.4 \mathrm{ml}$ of air. The stability of the foam and its microbubbles, as well as the distribution, is still under investigation [7].

Lastly, the meaning of "urgent" HD should be clarified. In fact, neither the pre-operative scores nor the blood tests (hemoglobin) were reported by Lisi et al [1].

Nevertheless, the authors did not declare the period and length of the enrollment. The latter may help to understand both the hospital overload and the incidence of HD in a tertiary referral center.

Foam ST is a promising, painless, repeatable, and lowcost procedure which does not involve sedation or the use of local anesthesia. It has a great potential for success especially when correctly used, i.e. for the treatment of patients with severe life-threatening bleeding, regardless of the degree of HD.

Furthermore, it can be used in elderly patients, with or without comorbidities, who could not undergo surgical treatment.

We agree with Lisi and colleagues that ST was extremely helpful during the COVID-19 outbreak as well as that this "new" treatment represents an arrow in every proctologist's bow. Further randomized controlled trials are needed.

\section{Compliance with ethical standards}

Conflict of interest The authors declare that they have no conflicts of interest.

Research involving human participants and/or animals This article does not contain any studies with human participants performed by any of the authors.

Informed consent Informed consent is not required.

\section{References}

1. Lisi G, Campanelli M, Grande S, Milito G, Grande M (2021) Sclerotherapy with $3 \%$ polidocanol foam for third- and fourthdegree hemorrhoids as "bridge treatment" during the COVID-19 pandemic in Italy. Int J Colorectal Dis. https://doi.org/10.1007/ s00384-021-03848-3

2. Gallo G, Sturiale A, De Simone V et al (2020) Deadlock of proctologic practice in Italy during COVID-19 pandemic: a national report from ProctoLock2020. Updates Surg 72(4):1255-1261. https://doi.org/10.1007/s13304-020-00860-0

3. Moser KH, Mosch C, Walgenbach M et al (2013) Efficacy and safety of sclerotherapy with polidocanol foam in comparison with fluid sclerosant in the treatment of first-grade haemorrhoidal disease: a randomised, controlled, single-blind, multicentre trial. Int J Colorectal Dis 28(10):1439-1447. https://doi.org/10.1007/s0038 4-013-1729-2

4. Ronconi M, Casiraghi S, Schieppati M (2019) EndoTHeF: endoluminal treatment of hemorrhoids with foam. Ann Colorectal Res 6(4):e86297

5. Lobascio P, Laforgia R, Novelli E, Perrone F, Di Salvo M, Pezzolla A, Trompetto M, Gallo G (2020) Short-term results of sclerotherapy with $3 \%$ polidocanol foam for symptomatic second- and third-degree hemorrhoidal disease. J Invest Surg 15:1-7. https:// doi.org/10.1080/08941939.2020.1745964

6. Fernandes V, Fonseca J (2019) Polidocanol foam injected at high doses with intravenous needle: the (Almost) perfect treatment of symptomatic internal hemorrhoids. GE Port J Gastroenterol 26(3):169-175

7. Critello DC, Pullano SA, Gallo G, Matula TJ, Fiorillo AS (2020) Low frequency ultrasound as a potentially viable foaming option for pathological veins. Colloids Surfaces A Physicochem Eng Asp 599:124919

Publisher's Note Springer Nature remains neutral with regard to jurisdictional claims in published maps and institutional affiliations. 